\title{
Redesigning care to meet national recommendation of four or more yearly clinic visits in patients with cystic fibrosis
}

\author{
A Berlinski, ${ }^{1,2} \mathrm{M} \mathrm{J}$ Chambers, ${ }^{1}$ L Willis, $^{1} \mathrm{~K} \mathrm{Homa}^{3}{ }^{3} \mathrm{G} \mathrm{Com}{ }^{1,2}$
}

- Additional material is published online only. To view please visit the journal online (http://dx.doi.org/10.1136/bmjqs2013-002345).

${ }^{1}$ Arkansas Cystic Fibrosis Care Center, Arkansas Children's Hospital, Little Rock, Arkansas, USA

${ }^{2}$ Pulmonology Section, Department of Pediatrics, University of Arkansas for Medical Sciences, Little Rock, Arkansas, USA

${ }^{3}$ Leadership Preventive Medicine Residency Program, Dartmouth Hitchcock Medical Center, Lebanon, New Hampshire, USA

\section{Correspondence to} Dr A Berlinski, Pulmonology Section, Department of Pediatrics, University of Arkansas for Medical Sciences, 1 Children's Way, Slot 512-17, Little Rock, AR 72202, USA; berlinskiariel@uams.edu

Received 22 July 2013

Revised 11 September 2013 Accepted 4 December 2013

\section{CrossMark}

\footnotetext{
To cite: Berlinski $A$, Chambers MJ, Willis L, et al. BMJ Qual Saf 2014;23: i42-i49.
}

\begin{abstract}
Cystic fibrosis (CF) is a chronic disease requiring patients to have frequent specialty healthcare visits to delay progression of lung disease, prevent and treat failure to thrive and initiate early interventions to prevent acute illness and complications. The CF Foundation recommends that patients have visits with the CF care team at least every 3 months. During participation in the CF Foundation Learning and Leadership Collaborative IV, the CF team at Arkansas Children's Hospital initiated quality improvement work with the aim to increase the percentage of patients attending clinic four or more times a year from 35\% in 2004 and $56 \%$ in 2005 (CF Foundation Registry data) to $90 \%$ or greater. We redesigned our scheduling system, rescheduled missed patient appointments in a timely fashion and created a process to monitor attendance.

This quality improvement work led to a sustained increase in the percentage of patients attending clinic visits four or more times a year reaching our goal of $90 \%$ in 2012. Improvements were also noted in the number of patients with body mass index/weight-for-length centile of 25 or greater, which could be related to more frequent clinic attendance.
\end{abstract}

\section{INTRODUCTION}

Cystic fibrosis (CF) is a complex lifethreatening genetic disease affecting approximately 30000 children and adults in the USA. ${ }^{1}$ The genetic defect results in abnormal production/function of the Cystic Fibrosis Transmembrane Regulator protein. This leads to multiorgan disease involvement affecting the lungs, sinuses, intestinal absorption, liver, pancreas and others. Lung disease is responsible for most of the mortality in this patient population. ${ }^{2}$
CF care centres that monitor patients more frequently and are more adherent to nationally recommended treatment guidelines exhibit higher lung function and better patient outcomes. ${ }^{3}$ Having patients come to clinic also allowed healthcare practitioners to obtain more respiratory cultures and perform pulmonary function testing. Although not reported by these authors, several others have demonstrated a direct correlation between good nutritional status, better lung function and overall outcomes. $^{4-6}$ This rationale supports the importance of nutrition improving lung health that translates to increased longevity. CF care centres that have a more aggressive approach to nutrition have reported better patient outcomes. ${ }^{7}$ Periodic evaluation of patients with $\mathrm{CF}$ allows for early detection and prompt intervention for several health problems. The latter includes failure to thrive, acquisition of new organisms (ie, Pseudomonas aeruginosa) in their sputum, decline in lung function, CF-related liver disease, CF-related diabetes and others. This concept has also been shown for other chronic diseases such as diabetes. ${ }^{8}$ The CF Foundation (CFF) guidelines recommend that patients over the age of 1 attend routine clinic appointments every 3 months and more frequently if needed. ${ }^{9}$

The Arkansas Children's Hospital $(\mathrm{ACH}) \mathrm{CF}$ Care Center is the only paediatric CF care centre accredited by the CFF in the state of Arkansas, with approximately 160 patients seeking care at the centre. The ACH CF care team responded to a request for applications from the CFF to participate in a quality improvement (QI) collaborative, CFF Learning and Leadership Collaborative IV (LLCIV), in 
2005. This collaborative was designed to accelerate the rate of improvement in CF care by training teams from centres in methods of QI. ${ }^{10}$ In January 2006, ACH CF Care Center leadership team members participated in LLCIV. Part of our work at the LLCIV was to examine our centre-level reports from the CFF Registry and determine where we wanted to focus our improvement work. Regular clinic attendance has been directly correlated with better patient outcomes. The percentage of patients attending clinic four or more times per year at our centre was at 35 in 2004 and 56 in 2005. Therefore, we thought an area for improvement was meeting the guideline of patients having four or more clinic visits per year. We developed Project 4 or More with the aim of increasing the percentage of patients attending clinic four or more times a year to $90 \%$ or greater. Having patients attend clinic four or more times per year was viewed as an opportunity to improve patient care. A nutritional pathway was also established later to consistently provide nutritional support to those patients who needed it.

\section{METHODS}

Our QI work involved intensive training and coaching done under the auspices of the LLCIV during an 18 -month period. A mentor/coach provided training and tools in QI methodology included in the $C F$ Action Guide. ${ }^{11}$ A local QI team consisting of the CF Center Director, CF Nurse Coordinator, social worker, respiratory therapist, nursing clinic director, statistician and parent representative participated in bimonthly conference calls with the coach and periodic webinars. In addition, a subgroup attended two 2-day off-site collaborative meetings. We completed assigned tasks including clinical microsystems analysis and established an internal dissemination strategy. Lessons learned from QI assignments were discussed in monthly CF Leadership Team meetings, which included hospital administration. Additionally, team members were provided access to information through postings in a shared computer drive and on an internal display.

We developed a fishbone diagram (figure 1) to document factors that were linked to patients attending clinic less than four times a year. These factors were External, Internal, Communication and Patient/ Family Perceptions, which were obtained from several sources. The medical charts of patients who attended clinic less than four times in 2005 were reviewed to determine common patterns of non-attendance in regards to age, gender, distance from centre, season, CF physician or insurance carrier. To obtain patients' and family members' perspective, they were asked to participate in a survey to identify issues relative to clinic attendance. Additionally, CF team members participated in brainstorming sessions to further identify other factors.

Another area of assessment was to understand how our scheduling system influenced patients having less than four visits per year. Process evaluation included how appointments were made, reminder letters/phone calls, a clinic time cycle analysis and number of CF appointment slots (see figure 2). In 2006, the transition to a centralised appointment scheduling centre was announced at our hospital, which provided further impetus to refine the internal process. Patients with CF were traditionally assigned to an individual paediatric pulmonologist who saw them during their

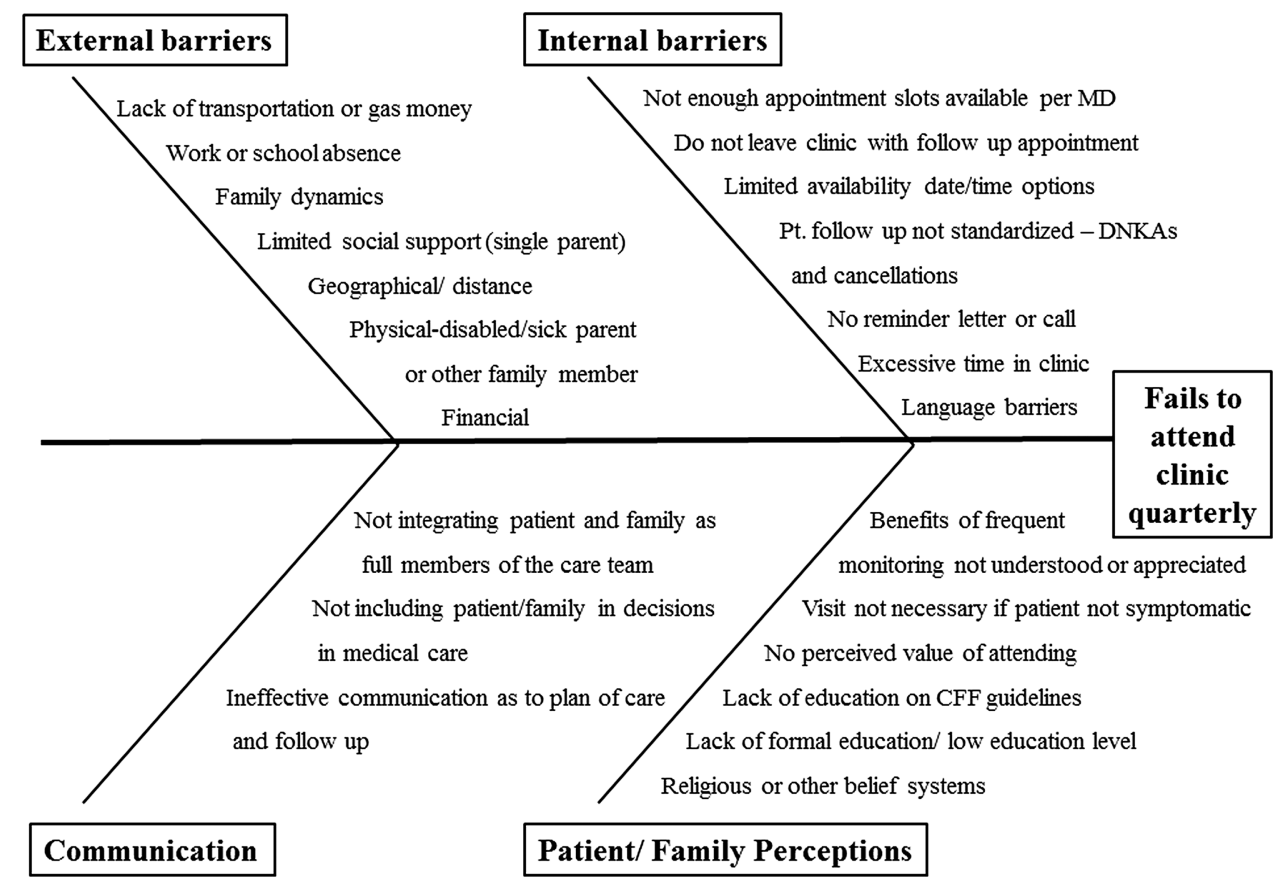

Figure 1 Fishbone diagram outlining barriers to clinic attendance at Arkansas Children's Hospital Cystic Fibrosis Care Center. 


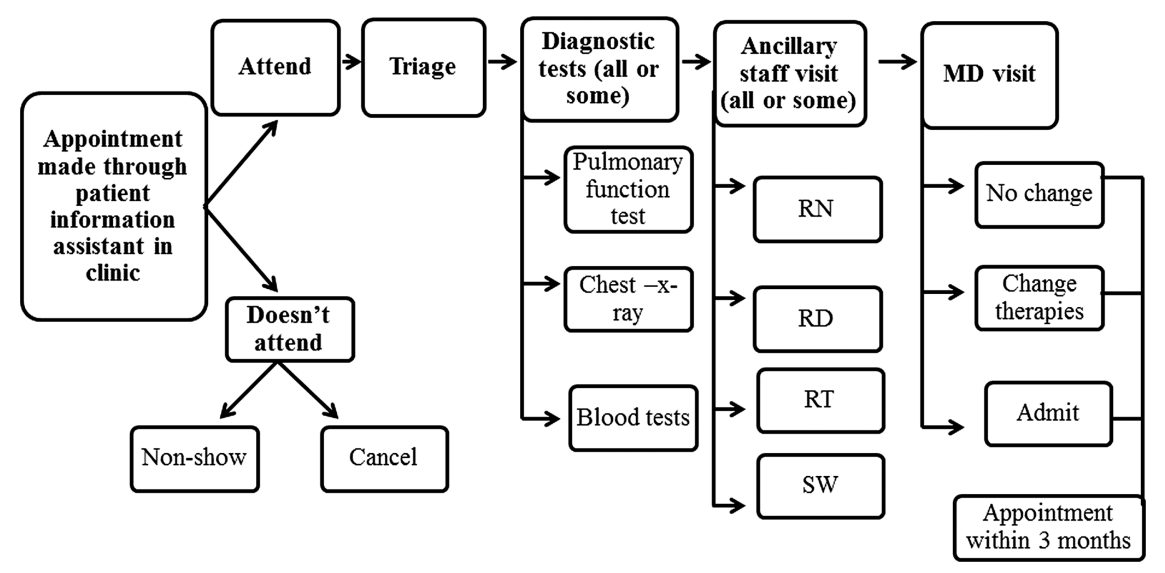

Figure 2 Flowchart of the clinic process at Arkansas Children's Hospital Cystic Fibrosis Care Center. Review of the clinic process uncovered that there was no standardised procedure to reschedule patients that did not keep their appointments.

scheduled clinic days along with other pulmonary patients. There was no plan to ensure appointment times were available in the schedule for the four or more visits, so specific CF appointment slots were developed. There was also no standard process to reschedule patients who missed their appointments.

Increasing patients' attendance to clinic four or more times/year was viewed as an opportunity to intervene with other QI work. Therefore, parallel to this improvement work focused on clinic attendance, a nutritional pathway was implemented during the last quarter of 2006 by some members of the CF team since good nutritional status has been associated with improved lung health. ${ }^{4-6} 12$ All patients were screened at each clinic visit and categorised in nutritional risk zones according to either their body mass index (BMI) centile for those 2 years old or older or their weight-for-length (W-L) centile for those younger than 2 years old (acceptable $>25$ th, nutrition risk 1025 th and urgent nutrition risk $<10$ th). Calculations were done using the BMI centile calculator supported by the Centers for Disease Control and Prevention (http://apps.nccd.cdc.gov/dnpabmi/) and weight-for-length calculator (http://www.uptodate. $\mathrm{com} /$ contents/calculator-cdc-nchs-infant-weight-forlength-percentiles-less-than36-months). Enzyme dosing was calculated at every nutritional encounter to monitor for underdosing or overdosing. Individualised nutrition plan and consultation were available upon physician's request. A standardised consultation form that included assessment for need for oral glucose tolerance test, bone densitometry and vitamin replacement was developed. The use of appetite stimulants and timing for gastrostomy tube placement were not included in the pathway and were left to individual physicians. Oral supplements were recommended for all patients with a BMI/W-L centile less than 25 th, and return clinic visits were scheduled within 4-6 weeks. Education was provided to patients and families regarding the importance of nutrition and their crucial role in monitoring and improving outcomes. This included more frequent clinic visits for weight checks, completing diet and stool records and ensuring intake of supplements and high-calorie, high-fat foods. Nutrition risk categories were revised in 2008 in concurrence with CFF goals for BMI/W-L >50th centile. ${ }^{12}$ The use of the pathway became standardised to include nutritional consult with a dietician for all patients $<50$ th BMI/W-L centile. The nutrition education booklet was revised to include the new nutrition categories and updated information regarding gastrostomy placement.

\section{Measuring the impact of the interventions}

The CFF Registry has a composite quality measure that reports the percentage of patients that had four or more visits per year, sputum culture and lung function testing. This composite measure reports only on enrolled patients and does not account for patients present less than four quarters. Therefore, we developed our own measure of clinical attendance, which was the number of patients seen per quarter (four visits per year) divided by the number of patients followed at the centre during the year. Patients newly diagnosed or who transferred care and were not at $\mathrm{ACH}$ all four quarters were excluded. Additionally, hospitalisations were not included as a clinic visit.

Statistical process control (SPC) charts were used to evaluate our QI work. SPC charts have the ability to distinguish between common cause and special cause variation. A measure in a stable system will vary over time, and the variation is just common cause or random variation. When something new enters the system, then the result will be a special cause variation. To determine whether clinic attendance changed significantly, we compared the annual rate from 2006 to 2012 using analysis of means (ANOM). ${ }^{13}$ ANOM for percent data contains the same formulas as the p-chart; however, there were not enough data points to evaluate the yearly data using a p-chart. The ANOM compared the annual rates against the overall rate and if the yearly rate of clinical 
attendance crossed either the upper or lower control limit then it was considered special cause in which something different entered the system that created a statistical significant result. Control limits were set at $2 \sigma$ (approximately to $2 \mathrm{SDs}$ ), which was at the 5\% risk level; however, traditionally limits are set at $1 \%$ risk, but we wanted to be sure to identify a significant result and thus we accepted a higher risk of type 1 error.

We also used an ANOM to determine whether there was an improvement in the annual percentage of patients with $\mathrm{BMI} / \mathrm{W}-\mathrm{L}<50$ th centile that had a consult with a dietician.

To determine whether the patients' nutritional status improved over time, we used a SPC X chart. ${ }^{14}$ Nutritional status measure was percentage of patients with a greater than 25 th centile BMI/W-L per quarter and was based on the highest centile BMI/W-L recorded during that period. The special cause variation rule that would represent a sustained improvement in patients' BMI/W-L centile would be eight or more consecutive points above the central line. The $\mathrm{X}$ chart displays quarterly measure of $\mathrm{BMI} / \mathrm{W}-\mathrm{L}$ centile over time, a centre line (the average of $\mathrm{BMI} / \mathrm{W}-\mathrm{L}$ centile measures) and upper and lower control limits that are similar to 3 SDs above and below the average.

The QI work was submitted to the University of Arkansas for Medical Sciences Institutional Review Board and was classified as exempt.

\section{RESULTS}

As a result of the initial assessment work from our participation in the LLCIV, communication and patient/family perception barriers were identified as important areas for improvement. To address patient and family involvement and improve communication, a family advisory board (FAB) was established in the spring of 2006 and family members participated in this QI work. ${ }^{15}$ The FAB's first newsletter described the importance of attending clinic appointments on a quarterly basis. Also, families started to receive a written summary of their clinic visit in the form of a CF Action Plan. ${ }^{16}$

The Appointment Center became operational in May 2007. They received 6-month rolling physician clinic calendars to allow creation of appointment slots. To address the scheduling barriers, an adequate number of appointment slots were created for each physician according to the number of CF patients under their care to ensure quarterly visits could be scheduled. Patients were assigned their follow-up appointment based on the information provided by the physician in a return appointment form completed at the end of the clinic visit. The system assigned appointments based on the physician's slot availability and did not provide families the option to choose a specific date and time. A decision tree outlining how to reschedule patients with CF was developed, and the appointment reminder system was revised (see figure 3). We established primary and secondary processes to reschedule patients who missed appointments. The CF office assistant received a daily automated email of appointment status from the previous day's clinic. The assistant then would send the list of patients who needed to be rescheduled to the Appointment Center to reschedule them within 2-3 weeks per the decision tree. If the patient could not be rescheduled within this timeframe, the Appointment Center would contact the nurse for that patient's doctor to assist in finding a new slot or overbook the clinic. Additionally, the CF Coordinator ran

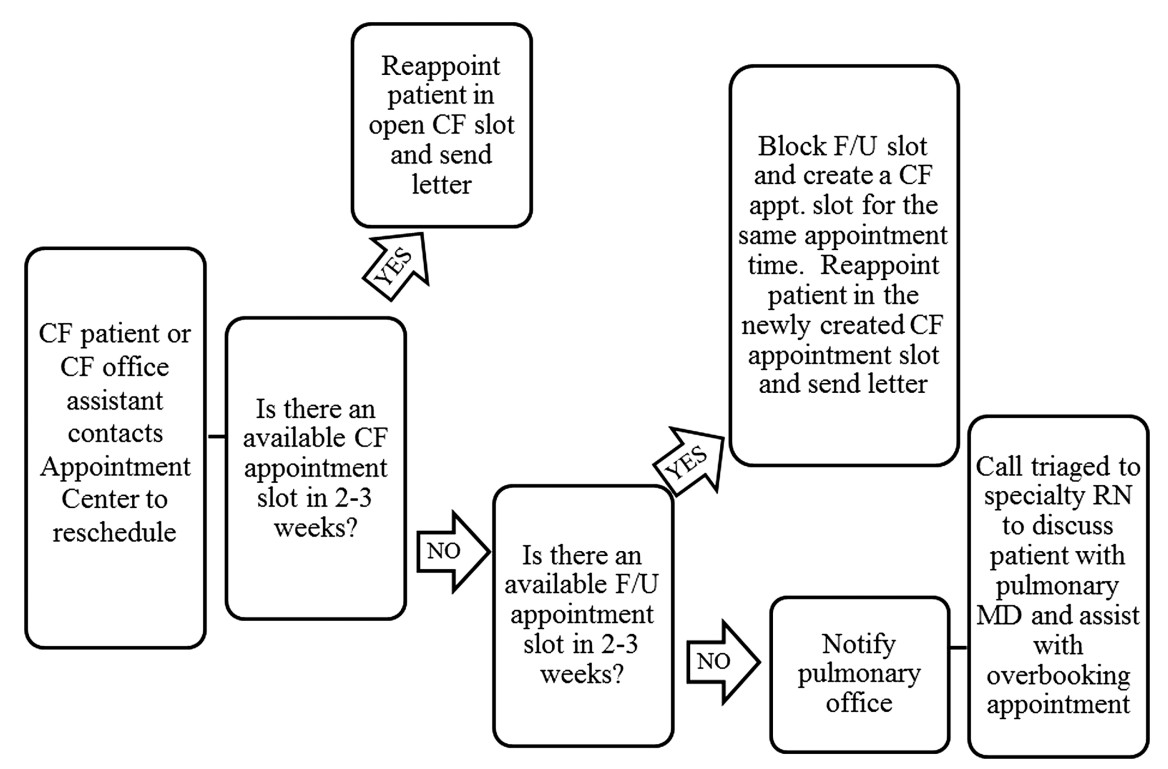

Figure 3 Centralised Appointment Center's Cystic Fibrosis (CF) rescheduling decision tree at Arkansas Children's Hospital CF Care Center. 
a monthly query from the CFF Registry (Port CF) about patients who were due a visit. After removing patients who were just seen or about to be seen, the finalised list was sent to the doctors and their nurses to contact and overbook patients immediately. To address the limitations of the existing monitoring systems, an internal database was created. The CF Social Worker tracked patient quarterly attendance and sent the list of patients not seen during the quarter to their doctors and nurses so that these patients would be rescheduled (see figure 4).

The online supplementary appendix 1 reports the timeline of different activities related to this improvement work from February 2006 to the end of the year in 2012.

\section{Outcomes of the interventions}

Between 2006 and 2012, 81\% of our patients had four or more visits per year. The ANOM chart (figure 5) of annual rates of patients having four or more visits shows the first year of 2006 of $72 \%$ was significantly lower than the succeeding years. From 2007 through 2011, the improvement was sustained and, in 2012, $90 \%$ of the patients had four or more visits, which was our goal and was significantly better than the overall rate. Figure 6 is another ANOM of annual rates of patients with BMI/W-L less than the 50th centile seen by a dietician. In 2005, it was 68\%, which was significantly lower than the succeeding years and significantly improved to $100 \%$ in 2009.
Figure 7 shows the $\mathrm{X}$ chart of percentage of patients having a greater than 25 th centile BMI/W-L. In 2007, $80 \%$ of the patients had a BMI/W-L greater than 25 th centile and in the third quarter of 2008 this increased to $82 \%$.

\section{DISCUSSION}

After implementation of QI interventions, the percentage of patients attending four or more clinic visits significantly improved, and in 2012 we met our goal of $90 \%$ of patients attending four or more times a year. A systematic approach to scheduling processes, timely rescheduling of patients who missed appointments and monitoring of attendance resulted in a significant increase in the number of patients who met the CFF national recommendation of four or more visits per year. This improvement was sustained over a 7-year period and is the standard of practice at our centre. One of the factors that we believe helped make the QI work successful was the internal monitoring and tracking of patients' clinic attendance, which is still continued in our clinic. Since the CFF Registry reports did not factor out patients who were not followed a full year (ie, diagnosed late in the year or who transferred care) and could not have attended four appointments, developing an internal outcomespecific database proved instrumental in achieving our goals. Additionally, other QI works led to an increase in the number of patients with 25 th centile $\mathrm{BMI} / \mathrm{W}-\mathrm{L}$ or greater as well as the number of patients with BMI/ W-L $<50$ th seen by a dietitcan. ${ }^{12}$

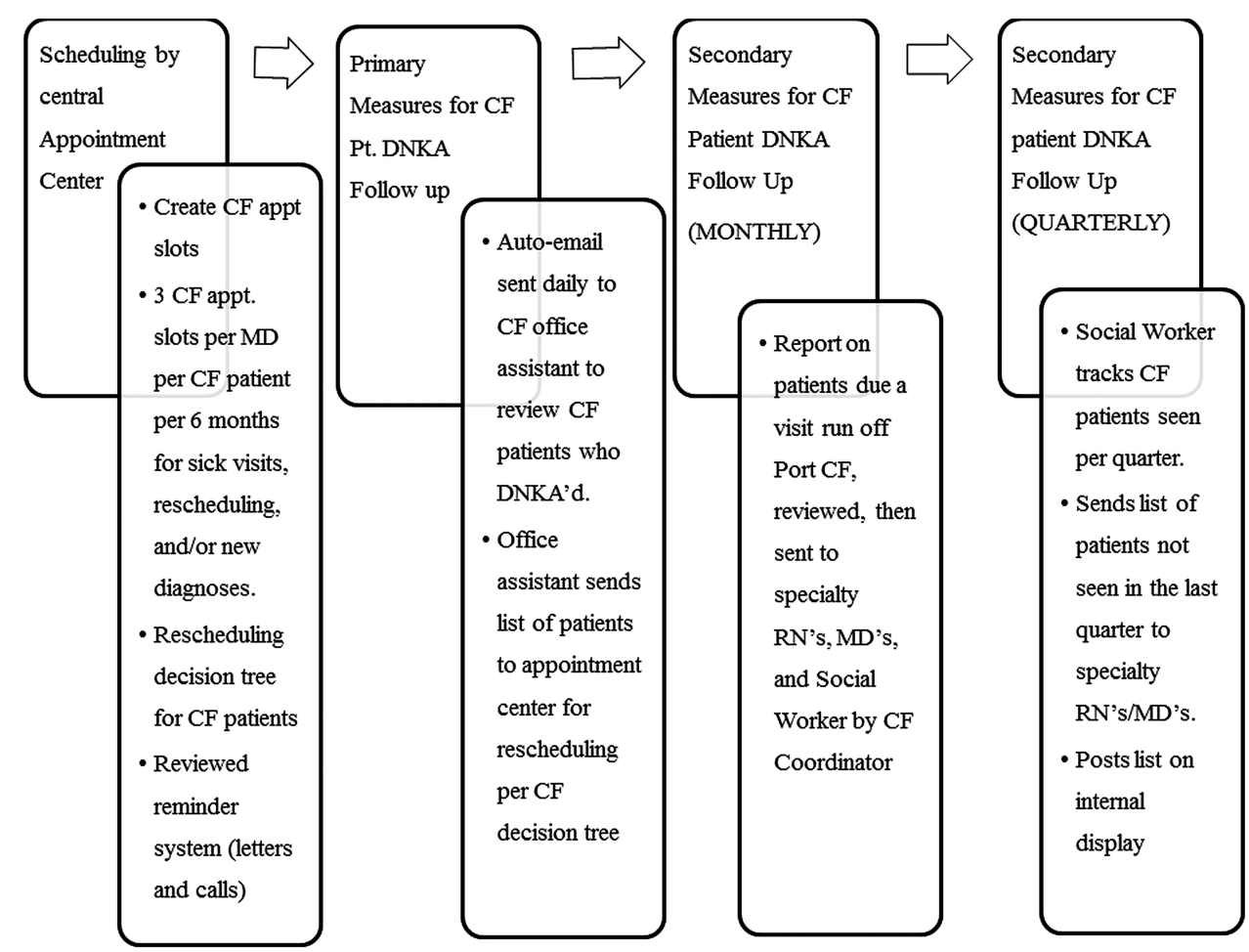

Figure 4 Standardised process to follow-up cystic fibrosis (CF) patients who 'do not keep appointments' (DNKA) to ensure four or more clinic visits per year at Arkansas Children's Hospital CF Care Center. 


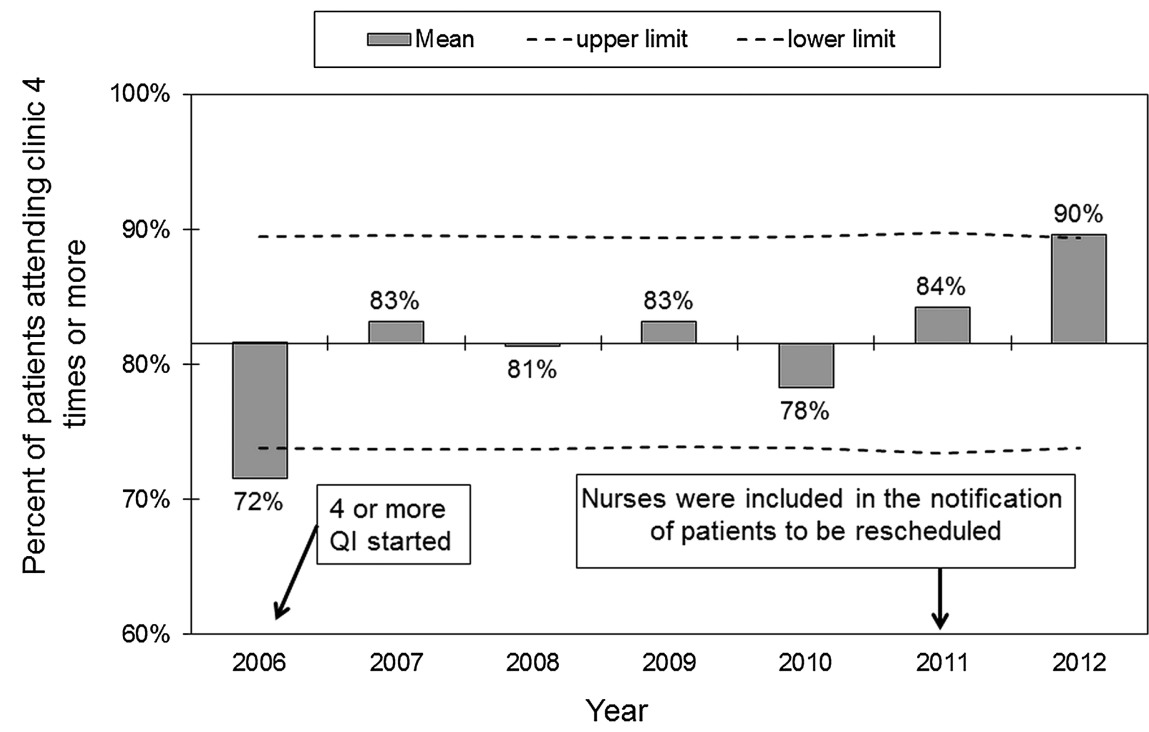

Figure 5 An analysis of means chart of percentage of patients per year attending cystic fibrosis clinic four times or more (20062012). Baseline line is the overall mean, each bar represents the individual data point and dashed lines are the upper and lower control lines set at $2 \sigma$. Any value outside these lines is considered statistically different.

Although there was a sustained improvement in the percentage of patients attending CF clinic four or more times per year, a marked improvement was noted in 2012. We speculate that the inclusion of the nurses in the notification of those patients that needed to be rescheduled was responsible for such an increment (see online supplementary appendix 1).

We viewed the increase in clinic attendance as an opportunity to monitor our patients more closely and to be able to implement other QI work. The nutritional pathway was one of the QI projects that were started.

The percentage of patients with BMI/W-L <50th seen by a dietician improved tremendously in 2009 after the nutritional consult changed from 'at physicians discretion' to mandatory. We speculate that nurses and nutritionists felt empowered to comply with this new requirement.

Although the increase in the percentage of patients with greater than 25 th centile for BMI/W-L from $80 \%$ to $82 \%$ might seem small, it represents a positive impact on a few more patients and provides more opportunities for improvement. We speculate that the increase in clinic attendance allowed for better implementation of the nutritional pathway and synergistically impacted the improvement gains in BMI/W-L. Our data are in agreement with Johnson et $a l^{3}{ }^{3}$ who reported that frequent monitoring among other interventions made possible due to patients being seen more in clinic was associated with improved outcomes in CF.

Our successful experience in developing internal processes to improve the percentage of patients being

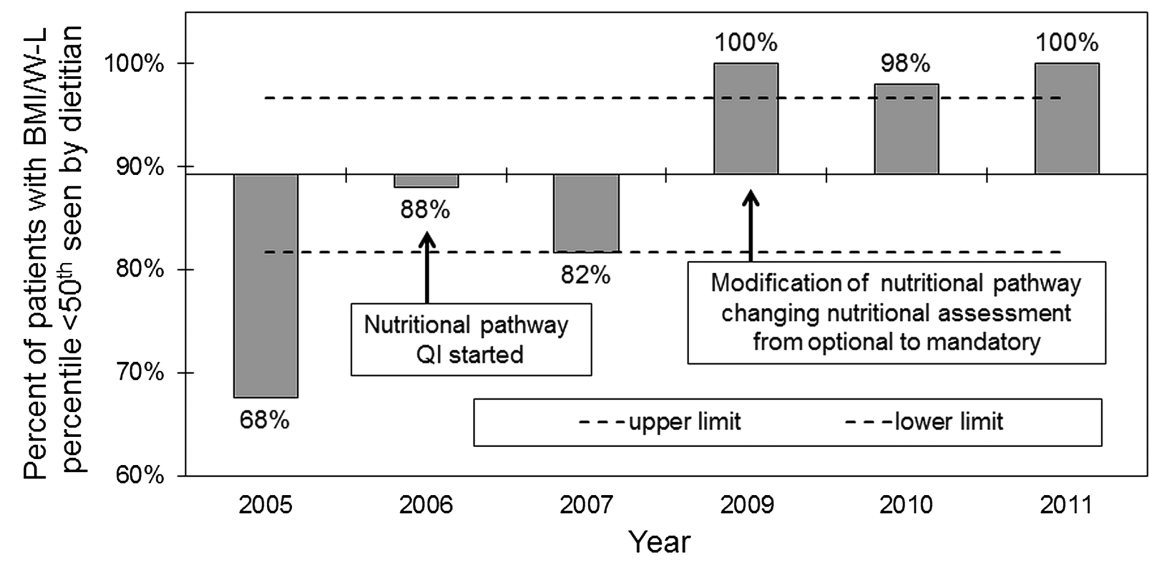

Figure 6 An analysis of means chart of percentage of patients with body mass index /weight-for-length <50th centile seen by dietician (2005-2011). Baseline line is the overall mean, each bar represents the individual data point and dashed lines are the upper and lower control lines set at $2 \sigma$. Any value outside these lines is considered statistically different. Data extracted from Cystic Fibrosis Foundation Registry. 2008 data were not collected due to local Institutional Review Board determination. 


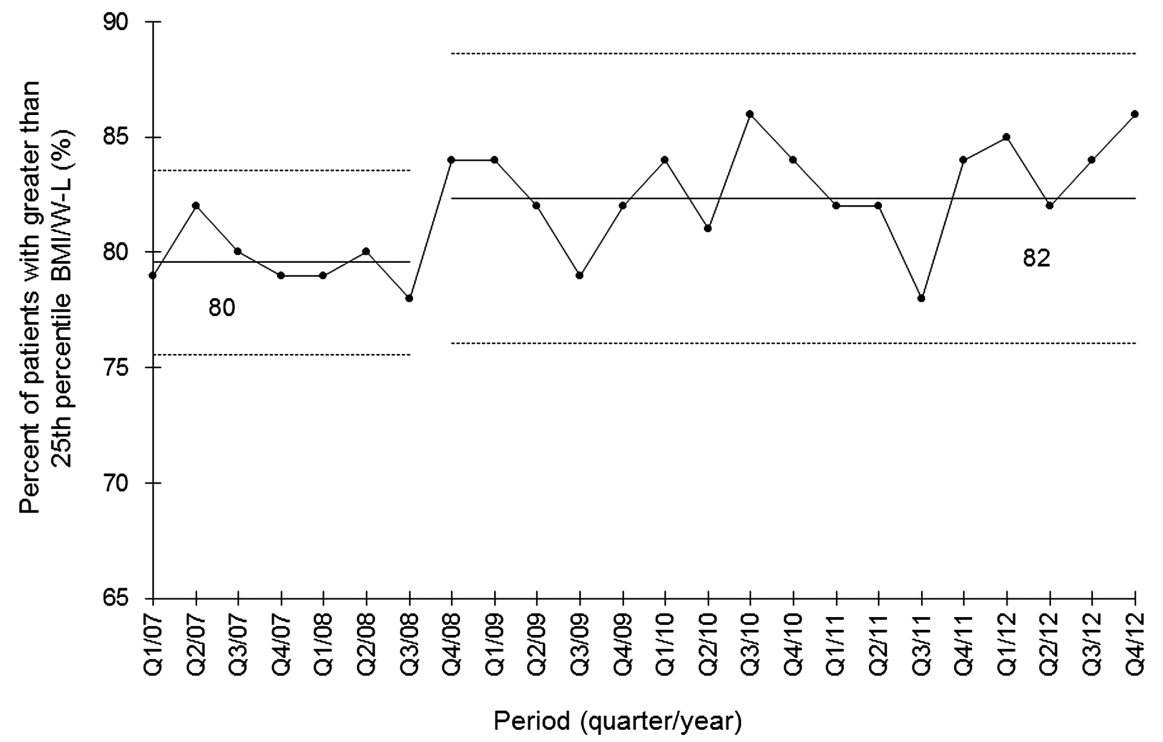

Figure 7 A statistical process control chart of nutrition assessment per quarter at Arkansas Children's Hospital Cystic Fibrosis Care Center (2007-2012). Control limits set at $3 \sigma$.

seen in the clinic four or more times a year can be adopted in other outpatient settings that require a specific number of scheduled visits/procedures over a period of time. Replication of these processes could be hindered by lack of personnel, lack of buy-in by the hospital administration and lack of patient/family involvement. A major challenge was maintaining consistency of the processes despite frequent personnel changes. Glitches in the current appointment reminder system sometimes result in late/missed letters and phone calls. Although we improved scheduling processes to ensure patients had appointments, there were still external barriers to attendance. Those barriers included rising fuel costs, transportation limitations, child care issues, missed workdays by caregivers and average low-income population.

Although changing processes of care can be met with resistance, our behaviours evolved by showing the value and the positive results that our process redesign brought. The overall acceptability of the need for QI crosses all disciplines. Hospitals have begun to strengthen their QI departments and are incorporating it in all areas of practice. Physicians are required to participate in QI work to obtain re-certification in their respective field. This improvement work was sustainable over time because of several factors such as Section Chief support, CF centre leadership commitment and the internalisation of the need for patients to be seen four or more times.

Many lessons were learned through our experience. We noticed our clinic attendance started to improve before the processes were fully implemented. We speculate this was due to the heightened awareness of our efforts by patients, families and our CF team. We learned that families are eager to have input and be involved in our centre processes. We found that this improvement work strengthened the interdisciplinary teamwork at our centre. We learned that QI work improves patient outcomes but requires institutional support, dedication and perseverance. Although the improvement work is labour intensive in a busy clinical setting, improved outcomes result in team motivation and pride of accomplishment.

An area of future improvement that is currently being evaluated is the patient's ability to schedule their return appointment before leaving clinic. Another possible improvement could be having a 12-month rolling physician clinic calendar to allow scheduling of all four appointments at one time. Additionally, availability of online scheduling would make the process more family centred. The creation of a hospital-wide electronic reminder system (text message/email) could potentially decrease nonattendance. This will require the availability of current and valid demographic information.

Lastly, participation in the LLCIV resulted in a positive culture change at the $\mathrm{ACH}$ CF Care Center regarding the use of QI methods to improve patient care. Our work was recognised by the CFF with the 2010 Annual CFF Quality Care Award.

\section{CONCLUSIONS}

Improvement work that focused on developing a systematic approach to scheduling and rescheduling resulted in a sustained improvement in the number of patients attending our CF clinic four or more times per year. In addition, the increased attendance facilitated the implementation of other QI work and improvement of an important patient nutritional outcome in $\mathrm{CF}$ care. 
Acknowledgements We are indebted to the families and patients with CF and to the CF care team at Arkansas Children's Hospital.

Contributors $\mathrm{AB}$ was responsible for the conception and design, analysis and interpretation of data and critical revision and final approval of the version to be published. MJC was responsible for the conception and design, drafting the article, critical revision and final approval of the version to be published. LW and GC were responsible for the conception and design, critical revision and final approval of the version to be published. $\mathrm{KH}$ was responsible for the analysis and interpretation of data and critical revision and final approval of the version to be published.

\section{Competing interests None.}

Ethics approval University of Arkansas for Medical Sciences IRB.

Provenance and peer review Not commissioned; externally peer reviewed.

\section{REFERENCES}

1 O'Sullivan BP, Freedman SD. Cystic fibrosis. Lancet 2009;373:1891-904.

2 Cystic Fibrosis Foundation Patient Registry. Annual data report to the center directors. Bethesda, MD: Cystic Fibrosis Foundation, 2010.

3 Johnson C, Butler SM, Konstan MW, et al. Factors influencing outcomes in cystic fibrosis: a center-based analysis. Chest 2003;123:20-7.

4 Konstan MW, Butler SM, Wohl ME, et al. Growth and nutritional indexes in early life predict pulmonary function in cystic fibrosis. J Pediatr 2003;142:624-30.

5 Steinkamp G, Wiedemann B. Relationship between nutritional status and lung function in cystic fibrosis: cross sectional and longitudinal analyses from the German CF quality assurance (CFQA) project. Thorax 2002;57:596-601.
6 McPhail GL, Acton JD, Fenchel MC, et al. Improvements in lung function outcomes in children with cystic fibrosis are associated with better nutrition, fewer chronic pseudomonas aeruginosa infections, and dornase alfa use. J Pediatr 2008;153:752-7.

7 Matel JL, Milla CE. Nutrition in cystic fibrosis. Semin Respir Crit Care Med 2009;30:579-86.

8 Morrison F, Shubina M, Turchin A. Encounter frequency and serum glucose level, blood pressure, and cholesterol level control in patients with diabetes mellitus. Arch Int Med 2011;171:1542-50.

9 Cystic Fibrosis Foundation Concepts in Care Consensus Conference. Preventative and Maintenance Care for the Patient with Cystic Fibrosis Vol 1, Section 2, page 6.

10 Marshall BC, Penland CM, Hazle L, et al. Cystic fibrosis foundation: achieving the mission. Respir Care 2009;54: 788-95.

11 Cystic Fibrosis Foundation. Action Guide for Accelerating Improvement in Cystic Fibrosis Care. 2006. http://www. clinicalmicrosystem.org/assets/materials/workbooks/cystic fibrosis_action_guide.pdf

12 Harden H, Com G. Maintaining the gains: ongoing assessment of quality improvement outcomes in nutrition status of CF patients [abstract]. Pediatr Pulmonol 2010;45(S33):405.

13 Homa K. Analysis of means used to compare providers' referral patterns. Q Manage Health Care 2007;16:256-64.

14 Amin SG. Control charts 101: a guide to healthcare applications. Q Manage Health Care 2001;9:1-27.

15 Chambers MJ, Willis L, Vaught F, et al. Improving patient and family involvement in the CF care center at Arkansas children's hospital through development of a family advisory board [abstract]. Pediatr Pulmonol 2006;41(S29):367.

16 Willis LM. Cystic fibrosis action plan: caregivers and healthcare team [abstract]. Pediatr Pulmonol 2010;45(S33):403. 